\title{
STUDIES OF ROTATIONAL-VIBRATIONAL DYNAMICS IN HIGHLY EXCITED HDO MOLECULE*
}

\author{
J. PYKA \\ Faculty of Chemistry, A. Mickiewicz University, Grunwaldzka 6, 60-780 Poznan, Poland
} (Received December 11,1990)

\begin{abstract}
The dynamics of highly excited HDO molecule is investigated. Calculations up to $J=40$ are performed for the bending-rotation Hamiltonian by applying the self-consistent (SC) methodology. Various aspects of rotation-vibration interaction are discussed. Centrifugal destabilization and stabilization of the molecular geometry is explained.
\end{abstract}

. PACS numbers: 33.10.Cs, 33.10.Jz

\section{Introduction}

Recent developments in Fourier transform and tunable laser techniques have revolutionized the study of molecular species by high-resolution infrared spectroscopy. It is now possible to achieve good separation of lines in spectra of highly excited molecules, but theoretical interpretation of these results becomes more and more difficult.

The standard perturbation theory generating effective rotational Hamiltonians for each vibrational state [1], which is generally used for interpretation and treatment of the rotational structure of vibrational levels, is valid only for the calculation of low rovibrational (RV) states of molecules. When highly excited RV states are taken into consideration, some serious problems arise. First of all in such states vibration-rotation interactions play a very important role. This is the reason why the perturbation approach cannot be applied in high-precision calculations. Moreover, this standard method is based on the assumption of small amplitude vibrations [2], therefore it must fail in the case of highly excited molecules when amplitudes of all internal motions are large.

*This research was supported by Grant II 15. 
The variational method enables one to a void some of these difficulties. Tennyson and co-workers [3-5] used it for calculations of high- $J$ states of triatomic molecules. However, in their approach, even in the case of such small molecules, diagonalization of very large secular matrices has to be performed. The physical interpretation of results is also very difficult as the wavefunctions are not obtained in a compact form.

This last remark is important because together with the necessity of accurate calculations of RV states there is a growing need for understanding how a molecule behaves under these conditions. The large number of classical and semiclassical investigations [6-12] is a consequence of the fact that, in contrast to the traditional quantum methods, classical mechanics provides means for gathering information about the approximate dynamics in high- $J$ regimes:

In this context the new self-consistent (SC) approach to the RV problem prom posed by Makarewicz $[13,14]$ se $m s$ to be an important step forward. In the frame of this theory an approximate separation of vibrational and rotational degrees of freedom can be performed and most of the important interactions between them are not neglected. What is also significant, the SC method is very effective and allows one to achieve accurate results even if modest means (personal computers) are applied.

The SC method together with new theoretical concepts, such as vibrational reduced density matrices, reduced local operators, vibrational and rotational population matrices [15], form a powerful tool for studying approximate dynamics of rotating-vibrating molecules.

Summing up, there is an effective quantum-mechanical method of calculation of the RV states which also can be applied in the areas reserved, till now, for the classical and semiclassical investigations. Studies of highly excited $\mathrm{H}_{2} \mathrm{O}$ [16] and $\mathrm{H}_{2} \mathrm{~S}$ [17] molecules are a good illustration of these new possibilities. In both cases the results of calculations enable one to distinguish several dynamical regions depending on the RV coupling and RV correlation. In some of these regions the molecular geometry is destabilized, in others stabilized with the equilibrium angle close to 90 . This stabilization is connected with the appearance of two equivalent local axes which are close to the bonds. The local axes break the symmetry $\mathrm{C}_{2 v}$, which is reflected in the cluster structure of the RV spectrum.

The purpose of this work is to investigate the most characteristic features of the bending-rotation spectrum and intramolecular dynamics of light triatomic molecules with $\mathrm{C}_{s}$ symmetry. As the well known and the most typical subject for study, HDO molecule is chosen.

The present paper is organized as follows. In Section 2 the brief discussion of the RV hamiltonian is presented. In Section 3 the method of calculations is. discussed. Results are presented in section 4.

\section{The bending-rotation model Hamiltonian}

The accuracy and efficiency of the methods of solving the RV Schrödinger equation depend considerably on the form of the RV IIamiltonian. Tennyson and 
co-workers $[3,4]$ derived the RV Hamiltonian by using the molecule fixed (MF) axes tied to the Jacobi type vectors. The kinetic energy operator is then given in the relatively simple form but the dimension of the Hamiltonian matrix is usually very large. This is so because these MF axes do not minimize the RV coupling which is very important in the highly excited RV states.

Recently, Makarewicz and Lodyga [18] derived a general Hamiltonian for a triatomic molecule introducing Eckart's idea [19] to the approach presented in [3, 4]. In the simplified bending-rotating molecular model they found the optimum MF axes which minimize the RV coupling. These axes eliminate the Coriolis term in the RV Hamiltonian, so they are the internal axes. It is however necessary to emphasize that the internal axes are optimum only when vibrational and rotational wavefunctions are determined from the SC equations [18].

In order to calculate the highly excited RV states of HDO molecule the bending-rotation Flamiltonian derived in [18] will now be applied. It means that from now vibration should be understood as a bending motion only. Figure 1 presents the RV coordinates of IIDO molecule based on Jacobi vectors $\boldsymbol{r}_{1}$ and $\boldsymbol{r}_{2}$. The internal $(x, y, z)$ axis system is rotated with respect to auxiliary $\left(x^{\prime}, y^{\prime}, z^{\prime}\right)$ axis system by the angle $\vartheta_{2}$ which is chosen in such a way that the Coriolis term in the RV Hamiltonian vanishes.

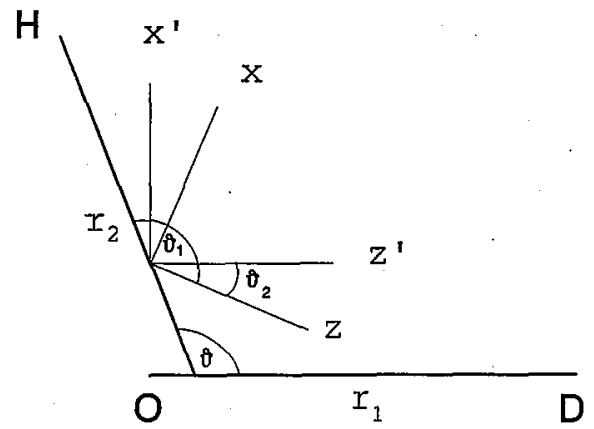

Fig. 1. The RV coordinates of HDO molecule based on the Jacobi vectors $\boldsymbol{r}_{1}$ and $\boldsymbol{r}_{2}, \boldsymbol{v}$, $r_{1}$ and $r_{2}$ are internal coordinates. The $z^{\prime}$ axis of the auxiliary axis system $\left(x^{\prime}, y^{\prime}, z^{\prime}\right)$ is parallel to the vector $r_{1}$, the $x^{\prime}$ axis lies in the plane $\left(r_{1}, r_{2}\right)$ and the $y^{\prime}$ axis is perpendicular to this plane. The internal axis system $(x, y, z)$ is rolated with respect to $\left(x^{\prime}, y^{\prime}, z^{\prime}\right)$ by the angle $\vartheta_{2}$.

The kinetic energy operator written in the internal axis system is given below:

$$
T(\vartheta, \varphi)=T_{\text {vib }}(\vartheta)+T_{\text {rot }}(\vartheta, \varphi),
$$

here $\vartheta$ is the bending coordinate and $\varphi$ represents the set of Euler angles. The vibrational energy operator is

$$
2 T_{\mathrm{vib}}(\vartheta)=I^{\mathrm{e}}\left[P_{\vartheta}^{2}-\hbar^{2}\left(1+1 / \sin ^{2} \vartheta\right) / 4\right],
$$


where $P_{\vartheta} \equiv-\mathrm{i} \hbar \partial / \partial \vartheta ; I^{\mathrm{e}}=I_{1}^{\mathrm{e}}+I_{2}^{\mathrm{e}}$. The values of $I_{i}^{\mathrm{e}}(i=1,2)$ are given in [18]. The rotational energy operator is

$$
2 T_{\mathrm{rot}}(\vartheta, \varphi)=G^{x x} J_{x}^{2}+G^{y y} J_{y}^{2}+G^{z z} J_{z}^{2}+G^{x z}\left(J_{x} J_{z}+J_{z} J_{x}\right) .
$$

The $G^{\alpha \beta}(\alpha, \beta=x, y, z)$ elements are as follows:

$$
\begin{aligned}
G^{x x} & =\left[I_{1}^{\mathrm{e}} \sin ^{2}\left(\vartheta_{1}\right)+I_{2}^{\mathrm{e}} \sin ^{2}\left(\vartheta_{2}\right)\right] / \sin ^{2}(\vartheta), \\
G^{y y} & =\left(I_{1}^{\mathrm{e}} I_{2}^{\mathrm{e}}\right) / I^{\mathrm{e}} \\
G^{z z} & =\left[I_{1}^{\mathrm{e}} \cos ^{2}\left(\vartheta_{1}\right)+I_{2}^{\mathrm{e}} \cos ^{2}\left(\vartheta_{2}\right)\right] / \sin ^{2}(\vartheta), \\
G^{x z} & =\left[I_{1}^{\mathrm{e}} \sin \left(2 \vartheta_{1}\right)+I_{2}^{\mathrm{e}} \sin \left(2 \vartheta_{2}\right)\right] / 2 \sin ^{2}(\vartheta),
\end{aligned}
$$

where $\vartheta_{1}=\vartheta+\vartheta_{2}$ and $\vartheta_{2}=-\left(I_{1}^{\mathrm{e}} / I^{\mathrm{e}}\right) \vartheta$. The angular momentum operators $J_{\alpha}$ are defined with respect to the internal axes.

The bending-rotation Hamiltonian looks as follows :

$$
H(\vartheta, \varphi)=T(\vartheta, \varphi)+V_{\mathbf{b}}(\vartheta)
$$

where the bending potential function is

$$
V_{\mathrm{b}}(\vartheta)=\sum_{n=1}^{4} V_{n} \cos ^{n}(\vartheta)-V\left(\vartheta_{\mathrm{e}}\right)
$$

The equilibrium value $\vartheta_{\mathrm{e}}$ and the potential parameters $V_{1}-V_{4}$ are taken from Table I in [18]. It has to be pointed out that there is an error in the table. The potential parameters are given in auxiliary units, not in $\mathrm{cm}^{-1}$, as it is written in the relevant caption. To receive values in $\mathrm{cm}^{-1}$, one has to multiply these numbers by the scaling factor equal to 33.71526 .

\section{Solution of the bending-rotating problem}

The self-consistent method of solving the RV Schrödinger equation is generally described in [13]. The SC equations for the vibrational and rotational functions in the case of the HDO molecule are shown in [18]. A detailed discussion of solving these equations is also presented there, so it will not be repeated here.

As a consequence of the above approach every RV state is approximately represented by the wavefunction:

$$
\Psi_{v r}^{\mathrm{SC}}(\vartheta, \varphi)=\Theta_{v r}^{\mathrm{SC}}(\vartheta) \Phi_{r v}^{\mathrm{SC}}(\varphi)
$$

where:

- $\Theta_{v r}^{\mathrm{SC}}(\vartheta)$ is the solution of the vibrational (bending) SC equation,

- $\Phi_{r v}^{\mathrm{SC}}(\varphi)$ is the solution of the rotational SC equation,

- $v$ is an approximate vibrational quantum number, 
- $r$ is a set of exact $(J, \tau)$ and approximate $\left(K_{\tau}\right)$ rotational quantum numbers.

The approximate quantum number $v$ is equal to the number of nodes in the vibrational function obtained by applying the Numerov-Cooley algorithm [20] to vibrational SC equation. The function $\Phi_{r v}^{\mathrm{SC}}$ is represented as a linear combination of the basis symmetrized functions $R_{K}^{J \tau}$ [21] whose coefficients are determined by diagonalizing the SC rotational energy matrix (see Appendix to the paper [18]). In general, $\Phi_{r v}^{\mathrm{SC}}$ are the eigenfurictions of the molecular inversion operator $E^{*}$ :

$$
E^{*} \Phi_{r v}^{\mathrm{SC}}=(-1)^{\tau} \Phi_{r v}^{\mathrm{SC}}, \text { where } \tau=0 \text { or } 1 .
$$

Therefore it is more convenient to introduce the approximate quantum number $K_{\tau}$ which will number these states and label their symmetry than to use traditional $K_{\mathrm{a}} K_{\mathrm{c}}$ notation.

The SC rotational-vibrational equations are coupled, so in order to solve one, the solution of the other has to be known. That is the reason why $\Theta^{\mathrm{SC}}$ depends not only on the $v$ number but also on the set of rotational quantum numbers and vice versa. To solve these coupled equations the very effective iteration technique is used [13].

In high-precision calculations the CI-like variational approach is proposed $[13,15-18]$. The construction of an SC/orthogonal (SCO) basis-function set is described in $[15,16]$. The "exact" RV wavefunctions $\Psi_{v r}^{\mathrm{CI}}$ can be expressed as the linear combinations

$$
\Psi_{v r}^{\mathrm{CI}}(\vartheta \varphi)=\sum_{v^{\prime} r^{\prime}} c_{v^{\prime} r^{\prime}} \Theta_{v^{\prime} r^{\prime}}^{\mathrm{SCO}}(\vartheta) \Phi_{v r^{\prime}}^{\mathrm{SCO}}(\varphi)
$$

This is the symbolic notation. Summing over $r^{\prime}$ means that for given $J$ and $\tau$ quantum numbers the sum goes over $K_{\tau}^{\prime}$. The $c_{v r}$ coefficients of Eq. (9) are determined by diagonalizing the matrix of the bending-rotation Hamiltonian formed in the SGO basis. Due to the symmetry this matrix is block-diagonal. For given $J$, we have two blocks depending on the $\tau$ value and their dimension is equal to the product of the number of vibrational functions by the number of adequate rotational functions. In the case of $\mathrm{C}_{2 v}$ molecules such as $\mathrm{H}_{2} \mathrm{O}$ or $\mathrm{H}_{2} \mathrm{~S}[16,17]$ the total Hamiltonian matrix could be (with the use of symmetry properties) factorized on four blocks which considerably reduces the numerical work. For HDO molecule we have to deal with matrices twice as large. It means for example, that in order to receive results of a reasonable accuracy $\left(0.01-0.1 \mathrm{~cm}^{-1}\right)$ for RV states with $J=40$, one has to diagonalize matrices of dimensions higher than 600 .

It is possible, however, to considerably lower this number. In paper [16] it was shown that there is no need to use the complete rotational basis in calculations. Usually half of this basis is enough to receive satisfactory results. It has to be emphasized that this conclusion is valid only when the rotational basis functions themselves are solutions of the SC rotational equation. If such a compact rotational SCO basis is used in linear combination (9), calculations of RV states of a highly excited HDO molecule become possible even on personal computer. In contrast however to the calculations of $\mathrm{H}_{2} \mathrm{O}$ [16] molecule, it is necessary to 
use a computer with the operation memory larger than $640 \mathrm{kB}$ ( all calculations presented in this paper were performed on a 32-bit unit). After the diagonalization of the Hamiltonian matrix, the correspondence between some predominant SC functions (included in the SCO basis) and the resulting $\Psi^{\mathrm{CI}}$ has to be found. This is the only way for the approximate quantum numbers, which are relevant in the SC approximation, to be assigned to the CI function. To solve this problem, the vibrational and rotational population matrices [15] are introduced. The values of their diagonal elements inform us about the contribution of a given $\Psi_{v r}^{\mathrm{SCO}}$ in the resulting $\Psi^{\mathrm{CI}}$, allowing some approximate classification. The rotational population matrices are also very important in choosing the proper rotation SC functions in the compact basis used for calculations of a desirable RV-CI state.

Sometimes, however, the SC functions are so mixed up in the resulting CI function, that such a simple analysis can not give a satisfactory answer. This, by the way, took place very often in the calculations of highly excited IIDO molecule. . In such a situation, the more complicated method was used. First, the model Hamiltonian

$$
H(C)=(1-C) H_{\mathrm{vib}}^{\mathrm{SC}}+C H
$$

was defined. Changing the parameter $C$ from 0 to 1 , one can pass from the SC Hamiltonian $H_{\text {vib }}^{\mathrm{SC}}$ to the bending-rotation IIamiltonian $H$. By calculating eigenvalues of $H(C)$ one can observe an evolution of each energy level and create the correlation diagrams linking $\Psi^{\mathrm{SCO}}$ to the $\Psi^{\mathrm{CI}}$. That is enough to make a unique assignment of $v r$ quantum numbers to a given $\Psi^{\mathrm{CI}}$.

\section{Results}

\subsection{Rotation Levels for $J \leq 20$}

The methodology outlined above was used for calculation of RV-CI states of IIDO molecule for $J$ number changing from 10 up to 40 . In this domain of $J$ two dynamical regions, depending on the strength of the RV coupling can be distinguished. In the first region, when $J \leq 20$ the rotational sublevels are organized as follows. When $J$ is even, the first $\bar{K}_{r}=0_{0}$, whereas when $J$ is odd, the first $K_{\tau}=0_{1}$. In the bottom of the energy levels we observe doublets of the states with the same $\tau$ symmetry. Then, as the $K_{\tau}$ grows these doublets begin to split. Higher on the energy ladder, the rotational sublevels with a different $\tau$ symmetry number, but the same $K$ number, come near to each other forming a new kind of doublets.

It is very illustrative to discuss the expectation values of the angular-momentum operators $J_{\alpha}$ and rotational constants $B_{\alpha}(\alpha=x, y, z)$ together with the energy values of these states. It is worth adding that effectiye rotational constants depend not only on the vibrational quantum number, but also on the rotational quantum numbers. This implies that effective moments of inertia change due to centrifugal interactions in rotating molecule. A detailed discussion of that result is given in $[13,14]$. 
Table I presents some of these values for chosen states with $v=0$ and $J=20$. All data given in this table were calculated with the wavefunctions of Eq.(9). Expectation values of nondiagonal rotational constant $B_{x z}$ and $\left\langle\left(J_{x} J_{z}+J_{z} J_{x}\right)\right\rangle$ are omitted in Table I because they do not give any additional information.

TABLE I

Energies, expectation values of angular momentum operators and rotational constants of chosen states of HDO molecule calculated for $v=0$ and $J=20$. Energies and rotational constants are given in $\mathrm{cm}^{-1}$. In accordance with the accepted model, $B_{y}$ is the constant for all states and equal to $6.326 \mathrm{~cm}^{-1}$.

\begin{tabular}{r|r|r|r|r|r|r|r}
\hline \hline$K_{\tau}$ & \multicolumn{1}{c|}{$\left\langle J_{x}^{2}\right\rangle$} & \multicolumn{1}{c|}{$\left\langle J_{y}^{2}\right\rangle$} & \multicolumn{1}{|c|}{$\left\langle J_{z}^{2}\right\rangle$} & \multicolumn{1}{c}{$B_{x}$} & \multicolumn{1}{c}{$B_{z}$} & $E_{\text {rot }}$ & \multicolumn{1}{c}{$E_{v r}^{\mathrm{CI}}$} \\
\hline $0_{0}$ & 25.53 & 390.24 & 4.23 & 9.22 & 22.92 & 2784.86 & 3476.27 \\
$1_{0}$ & 25.53 & 390.24 & 4.23 & 9.22 & 22.92 & 2784.86 & 3476.27 \\
$1_{1}$ & 76.40 & 331.50 & 12.09 & 9.21 & 22.94 & 3030.77 & 3724.39 \\
$2_{1}$ & 76.35 & 331.54 & 12.11 & 9.21 & 22.94 & 3030.79 & 3724.41 \\
$2_{0}$ & 127.64 & 273.85 & 18.51 & 9.20 & 23.01 & 3244.92 & 3946.16 \\
$3_{0}$ & 126.70 & 274.64 & 18.66 & 9.20 & 22.99 & 3245.43 & 3946.52 \\
$3_{1}$ & 185.29 & 212.34 & 22.37 & 9.17 & 23.18 & 3426.96 & 4138.43 \\
$4_{1}$ & 175.39 & 220.57 & 24.04 & 9.19 & 23.08 & 3432.59 & 4142.88 \\
$4_{0}$ & 258.84 & 139.70 & 21.46 & 9.11 & 23.54 & 3565.89 & 4288.56 \\
$5_{0}$ & 213.61 & 176.69 & 29.71 & 9.19 & 23.17 & 3598.79 & 4317.73 \\
$5_{1}$ & 301.61 & 96.18 & 22.21 & 9.08 & 23.71 & 3666.37 & 4395.39 \\
$6_{1}$ & 228.79 & 152.86 & 38.34 & 9.22 & 22.98 & 3758.99 & 4485.65 \\
$6_{0}$ & 274.28 & 112.36 & 33.36 & 9.16 & 23.30 & 3777.69 & 4508.37 \\
$7_{0}$ & 222.41 & 146.53 & 51.05 & 9.28 & 22.65 & 3930.28 & 4666.46 \\
$7_{1}$ & 231.77 & 138.28 & 49.94 & 9.27 & 22.72 & 3933.05 & 4669.80 \\
$\vdots$ & $\vdots$ & $\vdots$ & $\vdots$ & $\vdots$ & $\vdots$ & & $\vdots$ \\
$18_{1}$ & 64.31 & 44.72 & 210.97 & 10.31 & 18.87 & 6949.59 & 8143.79 \\
$18_{0}$ & 64.31 & 44.72 & 210.97 & 10.31 & 18.87 & 6949.59 & 8143.79 \\
$19_{0}$ & 51.91 & 28.22 & 339.87 & 10.38 & 18.64 & 7347.24 & 8587.18 \\
$19_{1}$ & 51.91 & 28.22 & 339.87 & 10.38 & 18.64 & 7347.24 & 8587.18 \\
$20_{1}$ & 40.28 & 9.93 & 369.79 & 10.44 & 18.43 & 7787.55 & 9055.19 \\
$20_{0}$ & 40.28 & 9.93 & 369.79 & 10.44 & 18.43 & 7787.55 & 9055.19
\end{tabular}

We :can see that the lowest states form doublets of close lying states with the same $\tau$ symmetry and different values of the $K$ number. The observation of the expectation values of the angular momentum operators reveals that the $\left\langle J_{y}^{2}\right\rangle$ is prodominant whereas the others are very small. In the classical limit, it means that for such states the angular momentum vector precesses around the axis which is almost perpendicular to the molecular plane (see Fig. 1). As the $\mathrm{K}$ number grows, $\left\langle J_{y}^{2}\right\rangle$ rapidly decreases, while $\left\langle J_{x}^{2}\right\rangle$ (rapidly) and $\left\langle J_{z}^{2}\right\rangle$ (slowly) increases. At the same time, effective rotational constant $B_{x}$ decreases and $B_{z}$ increases, while doublets of close lying rotational sublevels split, becoming separate states. 
In the states of this regime $\left\langle J_{x}^{2}\right\rangle$ is greater than both $\left\langle J_{y}^{2}\right\rangle$ and $\left\langle J_{z}^{2}\right\rangle$. Thus, in the classical limit, one should expect the angular momentum vector to move near the MF axis $x$. This conclusion is confirmed by the values of the rotational constants. When this kind of rotational motion takes place, the centrifugal forces make the molecule straighten, i.e. the equilibrium angle $\vartheta_{\mathrm{e}}$ is modified (increased). In this situation $B_{x}$ should decrease ( $B_{z}$ increase), and this is exactly what one can notice in Table I. Among the states of this regime the most distinct is the state $K_{\tau}=5_{1}$ for which $\left\langle J_{x}^{2}\right\rangle$ and $B_{z}$ reach their maxima, whereas $B_{x}$ is minimum. This is the separatrix state, which is the quantum analog of the separatrix trajectory on the classical rotational energy surface (CRES) introduced by Harter and Patterson [6].

The states surrounding the $K_{\tau}=5_{1}$ state are dispersed somewhat irregularly, but as the $K$ number grows a new kind of ordering emerges. Levels with the same value of $K$ number but different $\tau$ approach each other, forming new doublets eventually. At the same time $\left\langle J_{z}^{2}\right\rangle$ becomes the largest of all the expectation values of the angular momentum operators. This corresponds to the classical picture when a molecule rotates mostly around the $z$ MF axis. Centrifugal forces make the molecule bend then, so the $B_{z}$ effective rotational constant decreases, while $B_{x}$ increases (see Table I).

Summing up all these observations, we can say that the whole set of rotational sublevels can be divided into three groups characterized by the values of the expectation values of the angular momentum operators. The first one, is the $y$-group when the rotational sublevels form the doublets of the same $\tau$ symmetry. The rotational sublevels forming the $x$-group with the separatrix state as the most visible one, separate the $y$-group and the $z$-group, which also is formed by the set of doublets. The doublets of the $z$-group are characterized by the same $K$ and different $\tau$ numbers.

\subsection{Rolational levels for $J>20$}

The rotational energy levels structure of rigid and semirigid asymmetric rotors was explained by Harter and Patterson on the grounds of semiclassical mechanics. They introduced the classical rotational energy surface (CRES) which proved to be very helpful in understanding the qualitative features of RV spectra of such molecules [6,7]. The results presented in Table I and discussed above are in agreement with their findings. However, these results are typical only of IIDO molecule excited to medium values of the $J$ quantum number $(J \leq 20)$. When higher excitations are taken into account some new phenomena, generally connected with the RV coupling, perturb the clear picture presented above.

In his paper on semiclassical and quantum mechanical pictures of the RV motion of triatomic molecules, Makarewicz [22] pointed out that in nonrigid rotors RV interactions of rotational multiplets belonging to different vibrational states can perturb doublets splittings of close lying states or even can lead to the reversal of these doublets. In IIDO molecule such anomalous doublets in the $y$-group can be observed starting from $J=20$. 
TABLE II

Energies and diagonal elements of rotational population matrices $\left(R_{K K}\right)$ of the first four RV states of symmetry $\tau=0$, calculated for the chosen values of quantum number $J$.

\begin{tabular}{c|c|c|c|c|c}
\hline \hline \multicolumn{3}{c}{$J=20$} & \multicolumn{3}{c}{$J=24$} \\
\hline$E_{v r}^{\mathrm{CI}}$ & $R_{K K}$ & $E_{v r}^{\mathrm{CI}}$ & $R_{K K}$ & $E_{v r}^{\mathrm{CI}}$ & $R_{K K}$ \\
\hline & 0.556 & & 0.150 & & 0.000 \\
3476.27 & 0.444 & 4640.82 & 0.849 & 5298.98 & 0.999 \\
& 0.000 & & 0.001 & & 0.000 \\
& 0.000 & & 0.000 & & 0.001 \\
& $\vdots$ & & $\vdots$ & & $\vdots$ \\
\hline \multirow{3}{3}{3476.27} & 0.444 & & 0.849 & & 0.999 \\
& 0.556 & 4640.82 & 0.150 & 5298.98 & 0.000 \\
& 0.000 & & 0.000 & & 0.001 \\
& 0.000 & & 0.001 & & 0.000 \\
& $\vdots$ & & $\vdots$ & & $\vdots$ \\
\hline 3946.16 & 0.001 & & 0.002 & & 0.002 \\
& 0.002 & 5212.96 & 0.006 & 5921.63 & 0.006 \\
& 0.814 & & 0.501 & & 0.299 \\
& 0.178 & & 0.487 & & 0.686 \\
& $\vdots$ & & $\vdots$ & & $\vdots$ \\
\hline \multirow{3}{*}{3946.52} & 0.002 & \multirow{3}{*}{5213.01} & 0.006 & 5921.65 & 0.006 \\
& 0.178 & & 0.487 & & 0.686 \\
& 0.814 & & 0.501 & & 0.299 \\
& $\vdots$ & & $\vdots$ & & $\vdots$
\end{tabular}

In Table II the energies and rotational population matrices of first two doublets with $v=0$ and $\tau=0$, starting form $J=20$, are presented. One can notice that even for $J=20$ in the function of the lowest RV state, there is almost the same contribution of the second rotational SC state with $K_{\tau}=1_{0}$ as of the first with $K_{\tau}=0_{0}$. As number $J$ grows this tendency strengthens. When $J=24$, the first doublet is already reversed, i. e. in the wavefunction of type (9) which describes the lowest state, the predominant role is played by the second rotational SC function. The second doublet is strongly mixed up also. For $J=26$, both doublets are reversed. When the $J$ number grows higher doublets behave in the same way. Sometimes due to the RV coupling the whole situation gets normalized, i. e. the doublet which was reversed for lower $J$ becomes normal again for higher $J$. As a result, for the adequately high $J$ among the states of $y$-group, we can usually observe normal doublets as well as the reversed.

In Table III expectation values of the angular momentum operators and rotational constants for chosen rotational sublevels of the HDO molecule calculated 
for $v=0$ and $J=40$, are presented. In order to achieve the accuracy of 0.01-0.1 $\mathrm{cm}^{-1}$, fourteen vibrational and 30 rotational (compact basis) SCO wavefunctions werc used.

TABLE III Energies, expectation values of angular momentum operators and rotational constants of chosen states of HDO molecule calculated for $v=0$ and $J=40$. Energies and rotational constants are given in $\mathrm{cm}^{-1}$. In accordance with the accepted model $B_{y}$ is the constant for all states and equal to $6.326 \mathrm{~cm}^{-1}$. Reversed doublets of close lying states are distinguished by braces.

\begin{tabular}{r|r|r|r|r|r|r|c}
\hline \hline$K_{\tau}$ & \multicolumn{1}{|c|}{$\left\langle J_{x}^{2}\right\rangle$} & \multicolumn{1}{c|}{$\left\langle J_{y}^{2}\right\rangle$} & \multicolumn{1}{|c|}{$\left\langle J_{z}^{2}\right\rangle$} & \multicolumn{1}{c|}{$B_{x}$} & \multicolumn{1}{c|}{$B_{z}$} & \multicolumn{1}{c|}{$E_{\text {rot }}$} & $E_{v r}^{\text {CI }}$ \\
\hline $0_{0}$ & 50.88 & 1580.45 & 8.67 & 9.22 & 22.92 & 10629.60 & 11322.74 \\
$1_{0}$ & 50.88 & 1580 & 8.67 & 9.22 & 22.92 & 10629.60 & 11322.74 \\
$1_{1}$ & 152.26 & 1462.30 & 25.44 & 9.21 & 22.93 & 11130.47 & 11829.30 \\
$2_{1}$ & 152.26 & 1462 & 25.44 & 9.21 & 22.93 & 11130.47 & 11829.30 \\
$2_{0}$ & 242.92 & 1357.73 & 39.35 & 9.21 & 23.11 & 11505.27 & 12285.37 \\
$3_{0}$ & 242.92 & 1357.73 & 39.35 & 9.21 & 23.11 & 11505.27 & 12285.37 \\
$\vdots$ & $\vdots$ & $\vdots$ & $\vdots$ & $\vdots$ & $\vdots$ & $\vdots$ & $\vdots$ \\
$7_{1}$ & 760.88 & 794.76 & 84.35 & 9.11 & 24.73 & 12996.99 & 14111.17 \\
$8_{1}$ & 735.10 & 817.11 & 87.79 & 9.14 & 24.53 & 13005.82 & 14116.75 \\
$8_{0}$ & 945.85 & 614.39 & 79.76 & 8.97 & 25.68 & 13229.27 & 14373.43 \\
$9_{0}$ & 836.49 & 707.80 & 95.71 & 9.11 & 24.79 & 13277.18 & 14402.80 \\
$9_{1}$ & 1126.07 & 443.68 & 70.25 & 8.83 & 26.67 & 13396.70 & 14575.57 \\
$10_{1}$ & 895.17 & 637.74 & 107.09 & 9.12 & 24.80 & 13526.51 & 14672.12 \\
$10_{0}$ & 1082.77 & 474.46 & 82.78 & 8.91 & 26.31 & 13518.01 & 14754.36 \\
$11_{0}$ & 901.32 & 613.05 & 125.63 & 9.20 & 24.44 & 13779.23 & 14942.51 \\
$11_{1}$ & 987.95 & 540.78 & 111.27 & 9.10 & 25.12 & 13735.43 & 14960.25 \\
$12_{1}$ & 872.20 & 617.77 & 150.02 & 9.31 & 23.88 & 14037.01 & 15229.15 \\
$12_{0}$ & 894.44 & 600.37 & 145.19 & 9.29 & 24.02 & 14010.20 & 15229.06 \\
$13_{0}$ & 836.37 & 627.19 & 176.43 & 9.44 & 23.34 & 14290.97 & 15537.34 \\
$13_{1}$ & 843.82 & 620.32 & 175.87 & 9.44 & 23.26 & 14290.97 & 15532.80 \\
$14_{1}$ & 741.40 & 721.42 & 177.17 & 9.54 & 23.77 & 14163.05 & 15866.01 \\
$14_{0}$ & 809.85 & 624.47 & 205.68 & 9.58 & 22.66 & 14565.90 & 15859.23 \\
$\vdots$ & $\vdots$ & $\vdots$ & $\vdots$ & $\vdots$ & $\vdots$ & $\vdots$ & $\vdots$ \\
& & & & & & $\vdots$
\end{tabular}


TABLE III (cont:)

\begin{tabular}{c|c|c|r|c|c|c|c}
\hline \hline$K_{\tau}$ & $\left\langle J_{x}^{2}\right\rangle$ & $\left\langle J_{y}^{2}\right\rangle$ & $\left\langle J_{z}^{2}\right\rangle$ & $B_{x}$ & $B_{z}$ & $E_{\text {rot }}$ & $E_{v r}^{\mathrm{CI}}$ \\
\hline $32_{1}$ & 334.29 & 319.64 & 986.07 & 11.87 & 17.19 & 21105.41 & 24204.02 \\
$32_{0}$ & 334.29 & 319.63 & 986.08 & 11.87 & 17.19 & 21105.32 & 24204.02 \\
$33_{0}$ & 325.22 & 296.61 & 1018.16 & 11.84 & 17.20 & 21785.98 & 24913.28 \\
$33_{1}$ & 325.59 & 296.81 & 1017.60 & 11.84 & 17.21 & 21782.80 & 24913.28 \\
$34_{1}$ & 318.64 & 266.58 & 1054.79 & 11.81 & 17.08 & 22622.70 & 25661.25 \\
$31_{0}$ & 318.41 & 266.47 & 1055.12 & 11.81 & 17.08 & 22625.98 & 25661.25 \\
$35_{0}$ & 312.86 & 234.41 & 1092.73 & 11.78 & 16.99 & 23489.25 & 26450.16 \\
$35_{1}$ & 312.86 & 234.41 & 1092.73 & 11.78 & 16.99 & 23489.32 & 26450.16 \\
$36_{1}$ & 309.46 & 199.52 & 1131.04 & 11.73 & 16.89 & 24400.39 & 27279.99 \\
$36_{0}$ & 309.51 & 199.53 & 1130.96 & 11.73 & 16.89 & 24399.73 & 27279.99 \\
$37_{0}$ & 307.01 & 161.67 & 1171.32 & 11.68 & 16.79 & 25365.60 & 28150.18 \\
$37_{1}$ & 307.01 & 161.67 & 1171.32 & 11.68 & 16.79 & 25365.60 & 28150.18 \\
$38_{1}$ & 305.54 & 120.76 & 1213.69 & 11.62 & 16.70 & 26386.08 & 29060.78 \\
$38_{0}$ & 305.54 & 120.76 & 1213.69 & 11.62 & 16.70 & 26386.08 & 29060.78 \\
$39_{0}$ & 304.71 & 76.82 & 1258.48 & 11.56 & 16.59 & 27463.48 & 30011.53 \\
$39_{1}$ & 304.71 & 76.81 & 1258.48 & 11.56 & 16.59 & 27463.51 & 30011.54 \\
$40_{1}$ & 304.42 & 30.00 & 1305.59 & 11.50 & 16.47 & 28595.35 & 31002.28 \\
$40_{0}$ & 304.42 & 30.00 & 1305.59 & 11.50 & 16.47 & 28595.35 & 31002.28
\end{tabular}

At the first sight, the structure of rotational sublevels in Table III is similar to the one shown in Table I. Here one can also see two groups of doublets (some of them reversed) separated by the group of states. As before, the first $y$-group is characterized by the predominance of the $\left\langle J_{y}^{2}\right\rangle$ over the other expectation values of the angular momentum operators. In the $z$-group, this role is played by the $\left\langle J_{z}^{2}\right\rangle$. Among the states separating two types of doublets, the most visible is the scparatrix state $K_{\tau}=9_{1}$. In these states $\left\langle J_{x}^{2}\right\rangle$ is maximum, which corresponds to the classical picture when molecule rotates mostly around the $x$ MF axis. Due to the effect of centrifugal forces the molecule is straightened, so the $B_{z}$ effective rotational constant increases, while $B_{x}$ decreases (see Table III). To the $x$-group we can roughly assign states with $K_{r}$ from the range $8_{0} \leq K_{\tau} \leq 22_{0}$. These states spread somewhat irregularly but as the $K_{\tau}$ grows ( $\left\langle J_{x}^{2}\right\rangle$ decreases), we can notice that the states with the same $K$ but different $\tau$ come close to each other. In contrasi, however, with the results calculated for $J=20$ (Table I), now some other effects are clearly visible. Indeed, some pairs of states form reversed doublets (see Table III). These pairs are much more distant (several $\mathrm{cm}^{-1}$ ) than for doublets belonging to the $y$-group. This indicates that the reason for this effect is at much stronger RV interaction.

It is instructive to study how such a doublet comes into the existence. Let us look at two states with $K_{\tau}=14_{1}$ and $K_{\tau}=14_{0}$. The $14_{1}$ state should be the lower one. Instead, it lies about $7 \mathrm{~cm}^{-1}$ above the $14_{0}$ state. Even more drastic differences are observed in rotational characteristics of both states. The rotational 
energy of the $14_{1}$ state is smaller than such an energy of its partner on about 400 $\mathrm{cm}^{-1}$. All this can be explained assuming that the $14_{1}$ state is mixed with another state of higher vibrational quantum number and lower $K_{\tau}$ number. This mixing results from the RV interaction.

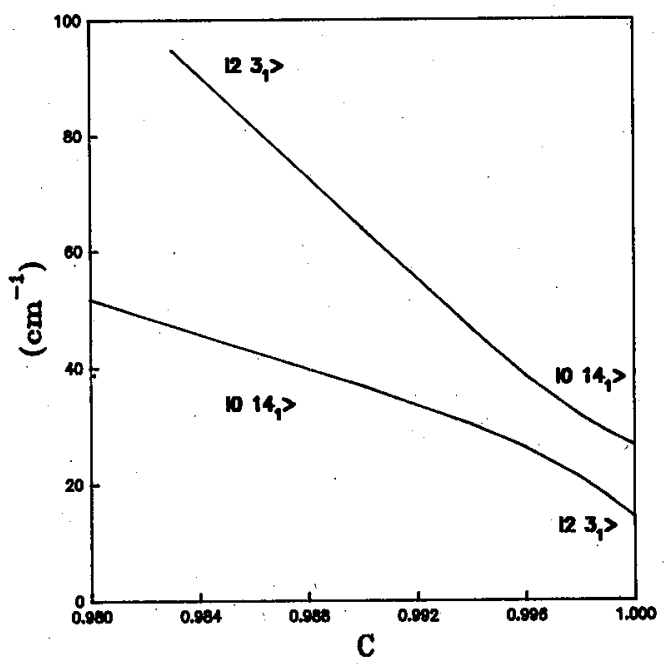

Fig. 2. Correlation diagram of the states $\left|014_{1}\right\rangle$ and $\left|23_{1}\right\rangle$ for $J=40$. In this symbolic notation the first number is the approximate vibrational quantum number $v$ while the second is the approximate quantum number $K_{r}$. The energies on this plot are given relative to the $E=15840 \mathrm{~cm}^{-1}$, which is taken as the zero point.

Using the Hamiltonian (10), we can find the state which mixes with the state 14. The results of such calculations for $J=40$ are presented in Fig. 2. For the sake of graphic presentation the evolution of only two states $\left(\begin{array}{ll}\mid 2 & 3 \\ 3_{1}\end{array}\right\rangle$ and $\left.\left|014_{1}\right\rangle\right)$ is shown in this figure. One can see that as the correlation parameter $C$ grows, both states come close to each other (the part of the correlation diagram for lower $C$ is omitted in Fig. 2 as not interesting). A closer examination of population matrices and other characteristics of both states tells us that as $C$ grows over 0.996 , both states start to mix. Furthermore, for $C=1$ the wavefunction of the upper state is a mixture of the $\left|23_{1}\right\rangle$ and $\left|014_{1}\right\rangle$ with the last prevailing. The lower state is also a mixture of both original states and $\left|23_{1}\right\rangle$ is predominant in this linear combination. Taking all this into account, we can approximately assign to the upper state the quantum numbers $v=0$ and $K_{T}=14_{1}$. However, we have to remember that the wavefunction of this state contains a contribution of the original $\left|23_{1}\right\rangle$ state. This is the reason why the expectation values of angular momentum operators, rotational constants and rotational energy calculated with this wavefunction are typical for the states with $K_{\tau}$ lower than $14_{1}$ (Table III). Also, the RV energy of this state is higher than for the state $\mid 014_{0}$. because it rises due to the contribution from $\mid 23_{1}$ ) state. As a result a reversed doublet 10 $\left.14_{1}\right\rangle$ and $\left|014_{0}\right\rangle$ emerges. 
The interactions between components of rotational multiplets belonging to different vibrational states appear often when HDO molecule is excited to a very high $J$ number. That is why in order to assign the approximate quantum numbers to most of the RV states, the correlation diagrams have to be constructed.

\subsection{Dynamics of the interacting rolational and bending motions}

The reduced vibrational density matrix $D_{v}(\vartheta)$ and the reduced local angular momentum operators introduced in [15] were already applied with success to the study of the RV dynamics of the $\mathrm{H}_{2} \mathrm{O}$ [16] and $\mathrm{H}_{2} \mathrm{~S}$ [17] molecules. Now, for the same purposes, they will be used in the investigation of the HDO molecule described by $v=0$ and $J=40$.

Plots of the $D_{v}(\vartheta)$, which is the probability density function calculated with the wavefunction $\Psi_{r v}^{\text {Cl }}$ [15], together with the centrifugal potential $V_{\mathrm{c}}(\vartheta)$ (defined and discussed also in [15]) are presented in Fig. 3. The states for which these plots are made were chosen as the most characteristic of a given dynamical region. In order to make the discussion more clear, plots of the effective potential $V_{\text {eff }}(\vartheta)$, which is the sum of the bending potential (6) and the centrifugal potential, are included.

The general examination of Figs. 3 leads to the conclusion that $D_{v}(\vartheta)$ packet changes its position and shape. The global shift of this function, without changing of its shape is an effect of a strong RV coupling. In that sense the coupling refers to "global" features of the molecular motion [15]. On the other hand, changes in $\dot{D}_{v}(\vartheta)$ shape are the manifestation RV correlation. This correlation can be understood as a kind of RV interaction leading to "local" changes of rotational and vibrational motions [15].

Plots of the reduced local angular-momentum operators [15] given in Fig. 3 are very helpful in studying that RV correlation in various rotational states. In the classical limit the reduced local angular operators $\tilde{J}_{\alpha}^{2}(\alpha=x, y, z)$ correspond to the instantaneous values (as the angle $\vartheta$ varies) of squares of the components of the angular momentum vector. It is necessary to emphasize that functions presented in Fig. 3 depenci on the angle between Jacobi vectors (Fig. 1). The interbond angle is smaller than the $\vartheta$ angle of about $6^{\circ}$. This difference is not important when general features of these functions are discussed. However, when a more detailed investigation is performed, this fact can not be neglected.

Figure 3(a) shows a situation typical of the states of $y$-group. The density function $D_{v}$ is localized around the angle $\vartheta=110.63^{\circ}$, which corresponds to the interbond equilibrium angle $104.62^{\circ}$ (see Table I in [18]). The centrifugal potential $V_{\mathrm{c}}(\vartheta)$ is approximately constant in the wide range of $\vartheta$, so the effective potential $V_{\text {eff }}(\vartheta)$ is in fact the bending potential with a constant added. At the same time, the curves $\tilde{J}_{\alpha}^{2}$ hardly change as the $\vartheta$ varies ( $\tilde{J}_{z}^{2}$ is negligibly small) so the RV correlation is small. This corresponds to the classical picture of a molecule rotating around the axis, which is almost perpendicular to the molecular plane. Moreover, the value of the $v$ angle does not have any influence on this motion.

As the $K$ number grows the situation changes. The $D_{v}(\vartheta)$ packet shifts in the direction of higher values of the angle $\vartheta$. For the separatrix state $K_{\tau}=9_{1}$ 
$648^{\circ}$

J. Pyka

(a)

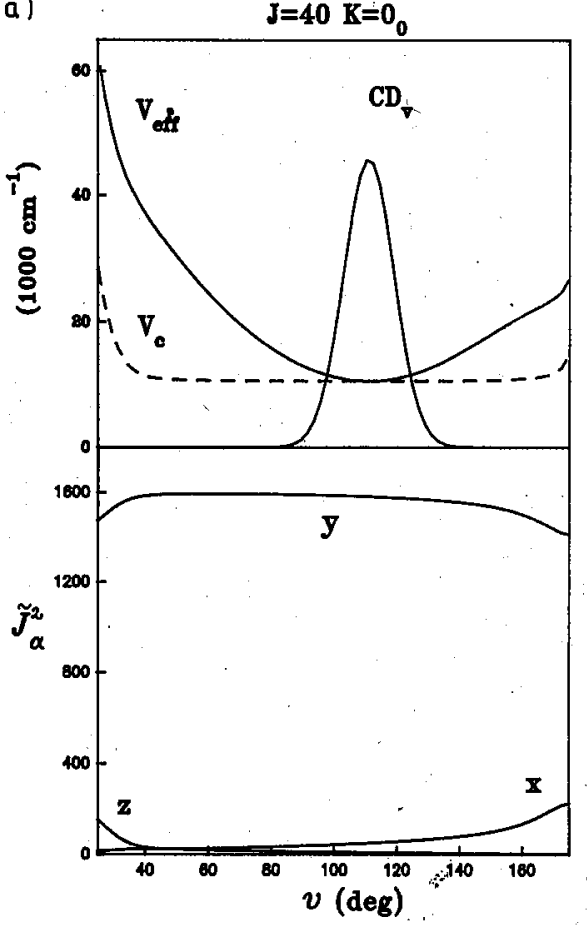

(c)

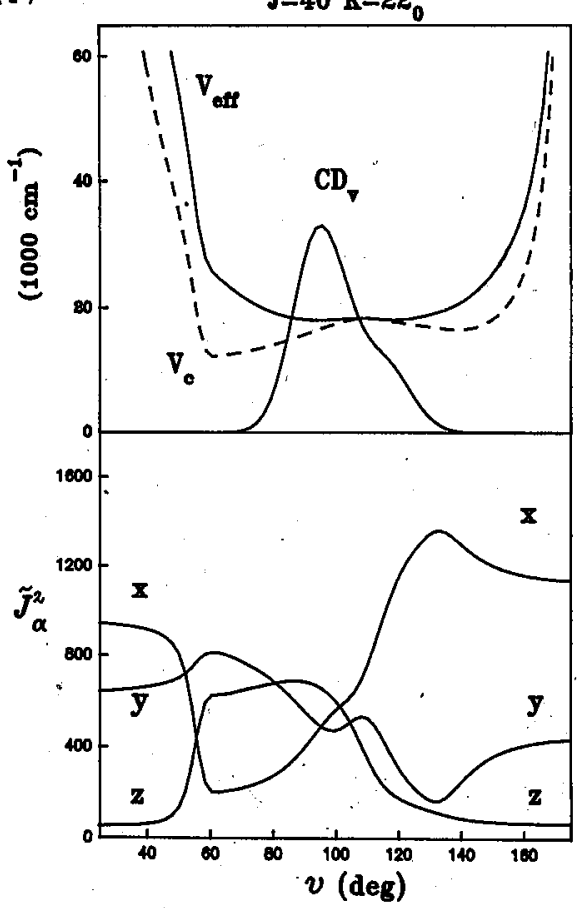

(b) $\quad J=40 \quad K=9_{1}$

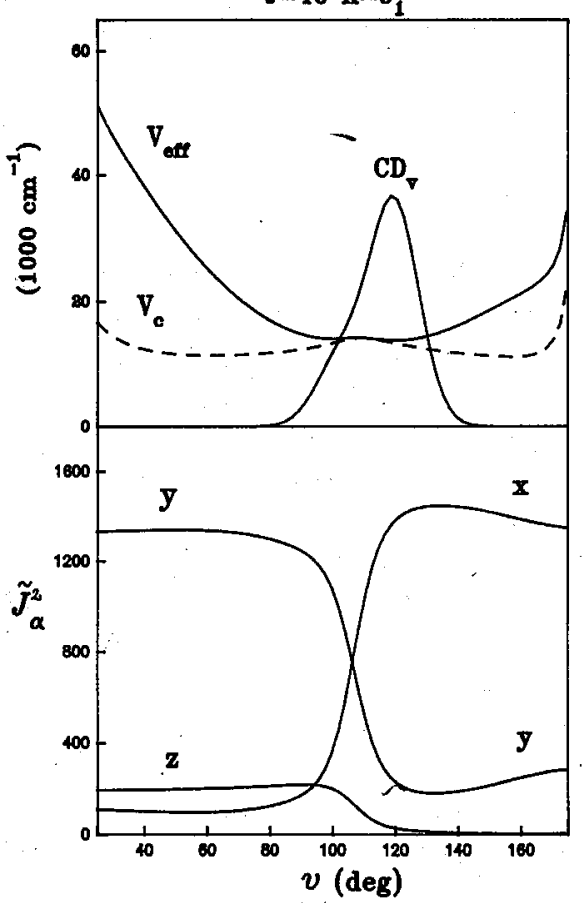

(d)

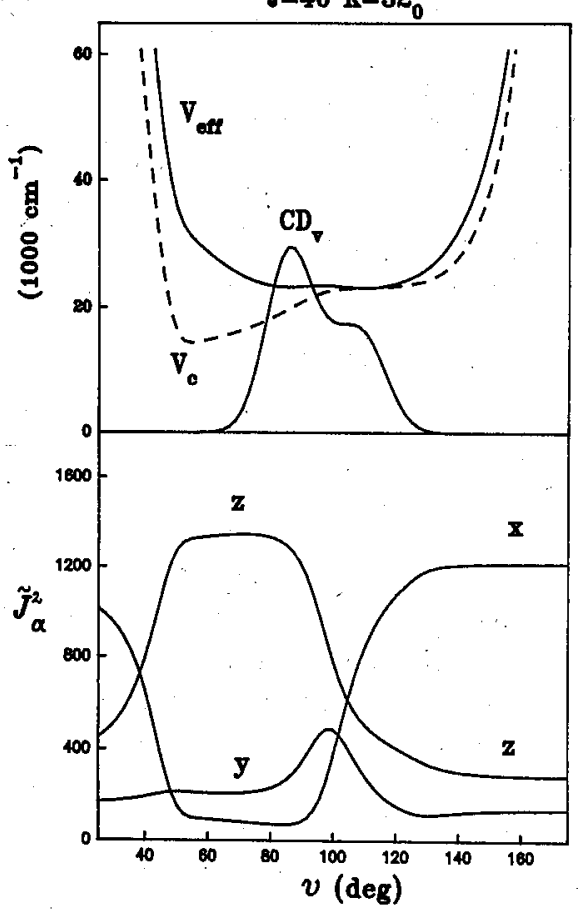




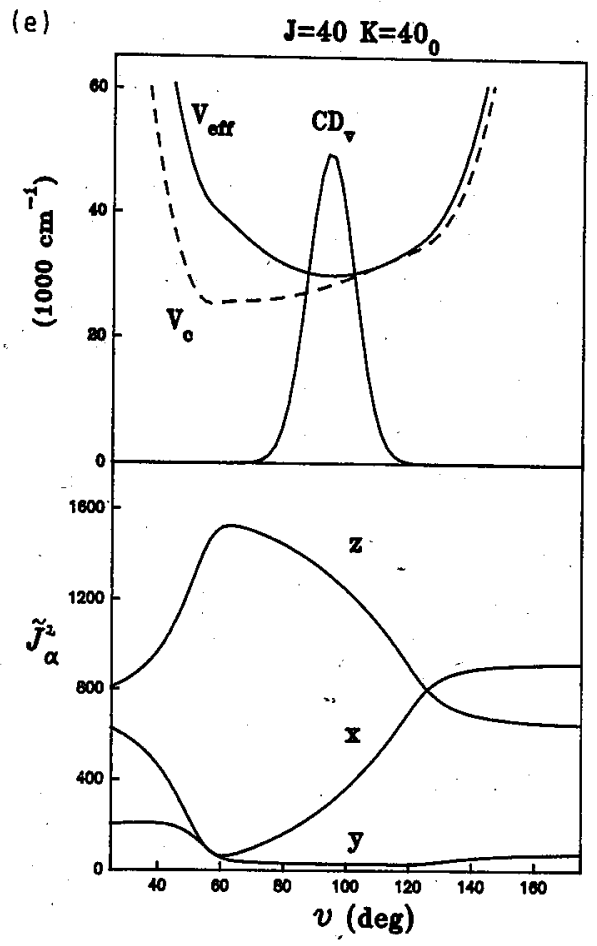

Fig. 3. Plots of vibrational density function $D_{v}(\vartheta)$, centrifugal potential $V_{c}(\vartheta)$ (dashed line), effective potential $V_{\text {eff }}(\vartheta)$, and local operators $J_{\alpha}^{2}(\vartheta)(\alpha=x, y, z)$ for states with $v=0$ and $J=40$; values of $K$ are given over the plots.For the graphical presentation purposes $D_{v}(\vartheta)$ is multiplied by the constant $C=8000 \mathrm{~cm}^{-1}$.

- (see Fig. 3(b)) the maximum of this function reaches $120^{\circ}$ (interbond angle is then about $114^{\circ}$ ). The shape of the centrifugal potential, which has two minima separated by a quite high potential barrier, now modifies the effective potential. As a result, it has also double minimum, even if these minima are placed at different angles than in centrifugal potential. This helps to explain the shift of the $D_{v}(\vartheta)$ packet. A detailed investigation of the effective potential for $K_{T}=9_{1}$ yields the following result: the left minimum placed at $100^{\circ}$ is $161 \mathrm{~cm}^{-1}$ deep, whereas the right mininum at $120^{\circ}$ is about $400 \mathrm{~cm}^{-1}$ deep. The density function is simply "pulled" inside the deeper well of the effective potential. In other words, the centrifugal forces make the molecule straighten (see discussion of Table III).

The double minimum in $V_{\mathrm{c}}(\vartheta)$ is the result of strong correlation between the vibrational and rotational motions (see Fig. 3(b)). First, when the molecule is strongly bent, the angular momentum vector $J$ is oriented almost along the MF $y$ axis. As the molecule straightens a dramatic change in the rotational motion occurs. In the range of $\vartheta$ angle changing from about $100^{\circ}$ to $115^{\circ} \tilde{J}_{y}^{2}(\vartheta)$ and $\tilde{J}_{x}^{2}(\vartheta)$ interchange. This corresponds to the classical picture when vector $J$ "jumps" from 
the MF $y$ axis to MF $x$ axis. The middle point where plots of both local operators intersect is about $108^{\circ}$. For this angle the barrier in the $V_{c}(\vartheta)$ occurs. This testifies to the direct relation between the RV correlation and the shape of the centrifugal potential.

The plots presented in Fig. $3(\mathrm{~b}-\mathrm{d})$ are characteristic of the wide range of states spread between $K_{\tau}=9_{1}$ and $K_{\tau}=32_{0}$. Due to the complicated character of the RV correlation, the shape of the effective potential is modified in such a way, that the left minimum becomes deeper than the right one. As a result, the density function shifts to the left (the bent configuration of the molecule is more probable). However, even if the left minimum is generally deeper than the right one, the difference between them is not big. For example, when $K_{\tau}=22_{0}$ the left minimum is $319 \mathrm{~cm}^{-1}$ deep, whereas the right one is about $283 \mathrm{~cm}^{-1}$ deep. In consequence, the $D_{v}(\vartheta)$ packet is localized mostly in the left well, but there is also some part of it which is localized in the right one. This brings about considerable change in the shape of this function. Besides the predominant left maximum, a small one on the right side of the potential barrier starts to rise. As a result, the $D_{v}(\vartheta)$ is spread over the wide range of the values $\vartheta$, changing from $70^{\circ}$ to $140^{\circ}$.

The case of the maximum bent molecule is shown in Fig. 3(d). However, the right maximum of the density function is now quite distinct, even if much lower than the right one. The $D_{v}(\vartheta)$ function shape is, in fact, typical of a molecule tunneling between two geometric configurations, through a low potential barrier (it is about $300 \mathrm{~cm}^{-1}$ for the state $K_{\tau}=32_{0}$ ).

Thus for the states between $K_{\tau}=9_{1}$ and $K_{\tau}=32_{0}$ we can observe a growing destabilization of the IIDO molecule geometry. It is caused by a complicated character of the rotational motion, which is strongly correlated with the vibration motion. This tendency reaches its culmination point for $K_{T}=32_{0}$.

As the $K_{\tau}$ grows over $32_{0}$, the left minimum in the effective potential quickly diminishes, while the right shifts in the direction of smaller angles, eventually becoming the single minimum. As a result, all signs of the geometry destabilization vanish and the maximum of density function shifts to the right, to the vicinity of $90^{\circ}$. Figure 3(e) shows this function for $K_{\tau}=40_{0}$. We can see that it is spread now over the range of about $30^{\circ}$ only. Its maximum $\vartheta$ angle is about $94.5^{\circ}$, which corresponds to the interbond angle $88.1^{\circ}$. At the same time plots of $\tilde{J}_{\alpha}^{2}(\vartheta)$ testify to the fact that the angular momentum vector precesses around a stable axis, which lies in the molecular plane and is almost parallel to the O-D bond. This conclusion is perhaps not obvious at the first sight, so a more detailed discussion will follow.

Factor $I_{1}^{\mathrm{e}} / I^{\mathrm{e}}$, which defines $\vartheta_{2}$ (see Eqs. (4) and Fig. 1) equals 0.3632 . When $\vartheta$ is small, $\vartheta_{2}$ is small too (although opposite directed). If there is the axis we are talking about, the $\tilde{J}_{z}^{2}(\vartheta)$ should be much bigger than the $\tilde{J}_{x}^{2}(\vartheta)$ and indeed it is. As the $\vartheta$ angle grows, $\vartheta_{2}$ also grows. This means, that $x, z$ axes of the internal axis system turn clockwise in order to remove Coriolis term (see Fig 1 ). If the $J$ vector precesses around the axis, which is close to the O-D bond, $\tilde{J}_{z}^{2}(\vartheta)$ should decrease while $\tilde{J}_{x}^{2}(\vartheta)$ should increase. This is exactly what one can observe in Fig. $3(\mathrm{e})$. When the angle $\vartheta$ comes close to $126^{\circ}$ both plots intersect. For this value of $\vartheta, \vartheta_{2}$ is about $45^{\circ}$ and the equality of both local operators confirm the existence 
of a stable rotation axis, which is almost parallel to the O-D bond.

This finding has its consequénces because rotation around such an axis stabilizes the bent configuration of the HDO molecule (see Fig. 4).

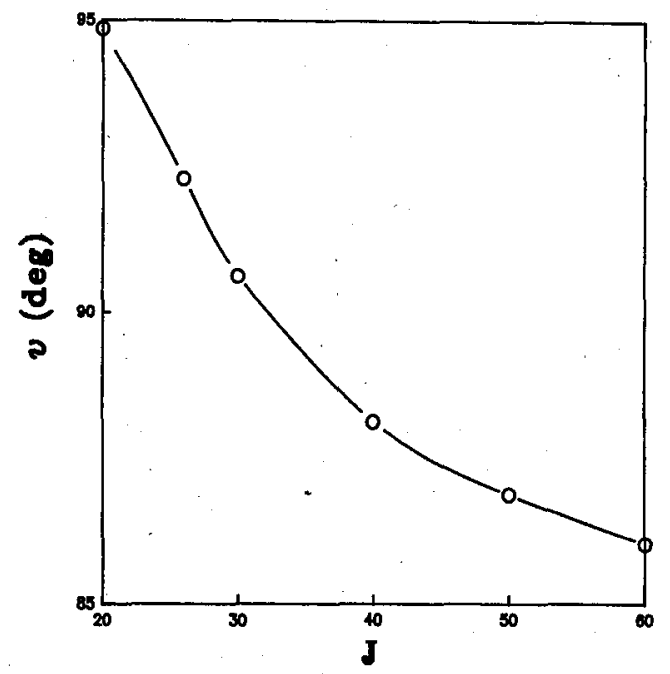

Fig. 4. The values of angles for which the vibrational reduced density function $D_{v}(\vartheta)$ reaches its maximum in states with $K=J$.

The values of angles for which the vibrational reduced density function $D_{v}(\vartheta)$ reaches its maximum in states with $K=J$, are plotted in Fig. 4. The angles are already transformed from the Jacobi-type vectors to the valence system. In that way, their values are equal to the values of the interbond angle. One can see that as the quantum number $J$ grows from 20 to 60 , the equilibrium angle tends to stabilize in the vicinity of $86^{\circ}$. Thus, for $K \approx J$ in highly excited IIDO molecule centrifugal forces stabilize the bent molecular configuration. The similar geometry stabilization was found in $\mathrm{H}_{2} \mathrm{O}$ and $\mathrm{H}_{2} \mathrm{~S}$ molecules $[16,17]$.

It should be added that, although the method of calculations presented in this paper enables one obtaining reasonable results for very high $J$ (up to $J=40$ ), solution of the RV problem for $J \geq 40$ becomes very difficult. There are two reasons for this. The first is a high density of the RV states, which complicates identification. The second, strictly numerical, is connected with the growing size of the matrices which need to be diagonalized. Only the states with either low $K$, or $K \approx J$ can be calculated with required accuracy for rotational quantum number $J$ higher than 40 . In that way, the results for $J=50$ and $J=60$ which are presented in Fig. 4, could be obtained.

At last one thing needs to be cleared out. During the discussion of the results given in Table III, the changes in values of the rotation constants were interpreted as consequence of the changes in the molecular geometry (when the molecule bends $B_{z}$ constant decreases while $B_{x}$ constant increases and vice versa). This 
interpretation is simple and straightforward, however it fails when states higher than $K_{\tau}=32_{1}$ are taken into consideration. Up to this state $B_{x}$ slowly grows while $B_{z}$ gets small, so the HDO molecule is becoming more bent. Above this state both $B_{x}$ and $B_{z}$ rotational constants decrease. Does it mean that the molecule at the same time becomes less and more bent?. It does not make sense. However, everything clears out when we take a closer look at the definition of the rotational constants.

Rotational constants $B_{\alpha}$ are calculated from the equation:

$$
B_{\alpha}=\frac{1}{2}\left\langle\Psi^{\mathrm{CI}}(\vartheta, \varphi)\left|G^{\alpha \alpha}(\vartheta)\right| \Psi^{\mathrm{CI}}(\vartheta, \varphi)\right\rangle_{\vartheta, \varphi}=\frac{1}{2} \int D_{v}(\vartheta) G^{\alpha \alpha}(\vartheta) \mathrm{d} \vartheta
$$

where $\alpha=x, y, z$.

It is now evident that the shape of the $D_{v}$ function determines the value of $B_{\alpha}$. Its shape for $K_{\tau}=32_{1}$ is almost exactly the same as for $K_{\tau}=32_{0}$ (see Fig. $3(\mathrm{~d})$ ). The maximum of this function lies on the left side (equilibrium angle is minimal) but the whole function is widely spread and there is a considerably high probability that the molecule is more straightened (destabilization of the molecular geometry).

As the $K$ number grows over 32 two processes start to take place. The first, which was discussed before, is connected with the shift of the maximum of $D_{v}$ to the right (eventually to the vicinity of $86^{\circ}$ ). The second is connected with the fact that at the same time the $D_{v}$ function is becoming less widely spread (for $K_{\tau}=40_{0}$ its width is only about $30^{\circ}$ ). Those two effects cause that $B_{x}$ and $B_{z}$ constants calculated from Eq.(11) decrease as the $K$ grows. Thus, even if the molecule straightens out as a whole, there can be the situation that the changes in rotational constants values do not reflect it directly.

\section{Summary}

In this paper the RV dynamics of the highly excited IIDO molecule has been studied. Calculations, based on the RV-SC theory, have been performed up to $J=40$. Strong interactions between rotational multiplets belonging to different vibration states were found to be the reason for the appearance of reversed doublets of rotational states. A detailed investigation of the vibrational density matrix and reduced local angular momentum operators plots has led to the conclusion that the RV coupling and RV correlation are the cause for the molecular geometry stabilization and destabilization.

\section{References}

[1] G. Amat, II.H. Nielsen, G. Tarrago, Rotation-Vibralion of Polyatomic Molecules, Dekker, New York 1971.

[2] E.B. Wilson, J.C. Decious, P.C. Cross, Molecular Vibrations, McGraw-Hill, New York 1955.

[3] J. Tennyson, B.T. Sutcliffe, Mol. Phys. 58, 1053, 1067(1986). 
[4] J. Tennyson, Comput. Phys. Rep. 4, 1 (1986).

[5] S. Miller, J. Tennyson, Chem. Phys. Lett. 145, 117 (1988).

[6] W.G. Harter, C.W. Patterson, J. Chem. Phys. 80, 4241 (1984).

[7] W.G. Harter, Comput. Phys. Rep. 8, 319 (1988).

[8] J.H. Frederic, G.M. McClelland, P. Brumer, J. Chem. Phys. 83, 190 (1985).

[9] J.H. Frederic, G.M. McClelland, J. Chem. Phys. 84, 876 (1986).

[10] J.II. Frederic, G.M. McClelland, J. Chem. Phys. 84, 4347 (1986).

[11] G.S. Ezra, Chem. Phys. Lett. 127, 492 (1986).

[12] B.I. Zhilinskii, I.M. Pavliczenkov, Opt. Spectrosc. 64, 688 (1988).

[13] J. Makarewicz, Mol. Phys., 61, 547 (1987).

[14] J. Makarewicz, J. Mol. Spectrosc. 130, 316 (1988).

[15] J. Makarewicz, Mol. Phys. 66, 113 (1989).

[16] J. Makarewicz, J. Pyka, Mol. Phys. 68, 107 (1989).

[17] J. Pyka, Mol. Phys. 70, 547 (1990).

[18] J. Makarewicz, W. Lodyga, Mol. Phys. 64, 899 (1988).

[19] C.F. Eckart, Phys. Rev. 47, 552 (1935).

[20] J.W. Cooley, Malh. Comput. 15, 363 (1961)

[21] P. Jensen, Comput. Phys. Rep. 1, 1 (1983).

[22] J. Makarewicz, Mol. Phys. 69, 903 (1990). 